\title{
Conditions for Moire Free Contact-Type 3 Dimensional Displays
}

\author{
Yoon-Chul Song \\ Korea Association of Information and Telecommunication Technology, Seoul, Republic of Korea \\ Vladimir V. Saveljev, Jung-Young Son* and Seok-Won Yeom \\ School of Computer and Communication Engineering, Daegu University, Kyungsan, Republic of Korea \\ Yu. A. Vashpanov \\ ECE Dept., Hanyang University, Seoul, Republic of Korea
}

(Received April 10, 2008 : revised June 1, 2008 : accepted June 10, 2008)

\begin{abstract}
The superposing angle of the viewing zone forming optics and the display panel in the contact type 3 dimensional imaging systems for minimizing moirés is found for a rectangular shape pixel with different aspect ratios. The angles are $26.2609^{\circ}$ for square shape pixels and $13.9858^{\circ}$ for the rectangular with aspect ratio 2 . These angles result in the moirés with the smallest period for the respective aspect ratio. The effectiveness of the angles is also experimentally demonstrated.
\end{abstract}

Keyword: Moire, contact-type 3 dimensional imaging system, viewing zone forming optics, display panel, superposing angle, moiré minimization

OCIS codes : (110.0110) Imaging systems; (110.6880) Three-dimensional image acquisition; (110.4280) Noise in imaging systems

\section{INTRODUCTION}

Moire is a natural interference phenomenon appearing whenever a transparent sheet with a regular pattern is attached on the top of a sheet/plate with a periodic pattern[1]. It has been used to measure various physical characteristics of various materials, device and systems [2]. Since the display panel in a contact-type multiview 3 dimensional (3-D) imaging system[3,4] basically consists of two optical layers with highly periodic patterns represented by a flat panel display and transparent viewing zone forming optics, moirés are inherent to the system. The moirés color the displayed 3-D image differently and occasionally making the image unrecognizable. However, moirés are not always bad, because they can give valuable information to estimate the degree of alignment between the viewing zone forming optics and the display panel[5]. But their presence on the display panel is highly undesirable because they deteriorate the quality of images displayed on the panel. Furthermore, moires appearing at the 3-D imaging system

\footnotetext{
*Corresponding author: sjy@daegu.ac.kr
}

are worse than those at a plane imaging system because they vary with change in viewing distances and directions. This is due to the finite thickness of the viewing zone forming optics. The pitch of the elemental optics in the viewing zone forming optics relative to the pixel pitch of the display panel is changing as the distance and direction change. Hence moirés become more cumbersome and recognizable because of the variation. Furthermore, the moirés can be minimized but cannot be completely eliminated unless the thickness of the viewing zone forming optics is negligible, and its pitch is exactly the same as that of the display panel.

One approach to minimizing the moires is to reduce the regularity of the viewing zone forming optics' and display panel's pitch relative to each other. This can be done by aligning the vertical axis of the elemental optics in the viewing zone forming optics to have a certain angle (here it is referred as an overlapping angle) with the vertical pixel line of the display panel $[6,7]$. The angle was calculated as $26.261^{\circ}$ for the case when the shapes of both the elemental optics and a pixel in the panel are squares, without regard to their 
sizes. This angle can be applied to most display panels because their pixels are square. But for the high resolution display panel from IBM[8], the pixel is rectangular. So the overlapping angle cannot be applied in this case.

In this paper, the overlapping angles between a viewing zone forming optics and a display panel having a rectangular shaped pixel to minimize the moirés, are calculated by varying the aspect ratios of the rectangular pixels.

\section{MATHEMATICAL FORMULATION OF MOIRÉ FORMING}

Moirés appearing when a display panel and viewing zone forming optics can be calculated by assuming that the pixel pattern in the panel and the elemental optics pattern in the optics can be approximated as 2 dimensional (2-D) wire grids with different pitches and aspect ratios. A wire grid can be approximated as 2-D line gratings and represented mathematically as[5],

$$
G_{W}=\frac{1}{4}\left(1+\cos \vec{k}_{x} \cdot \vec{x}\right)\left(1+\cos \vec{k}_{y} \cdot \vec{y}\right)
$$

where $G_{W}$ represents the grating function of the 2-D wire grid, 1 the average value term and $\vec{k}_{x(y)}$ is wave number in $x(y)$ axis direction which is represented as $2 \pi / P_{x(y)} . P_{x(y)}$ is the pitch of the wire grid in $x(y)$ axis direction. When two wire grids identified as 1 (elemental optics pattern in the viewing zone forming optics) and 2 (pixel grid in the display panel), are superposed together, it is represented as [6],

$$
F=\frac{1}{16}\left(1+\cos \vec{k}_{1 x} \cdot \vec{x}_{1}\right)\left(1+\cos \vec{k}_{1 y} \cdot \vec{y}_{1}\right) \times\left(1+\cos \vec{k}_{2 x} \cdot \vec{x}_{2}\right)\left(1+\cos \vec{k}_{2 y} \cdot \vec{y}_{2}\right)
$$

In Eq. 2, $F$ represents moirés when two wire grids are superposed together. When it is assumed that $\sigma(\sigma \geq 1)$ and $\rho(\rho \geq 1)$ represent the aspect ratio of the $1^{\text {st }}$ grid and the pitch ratio of the two grids, respectively, the scalar products of the cosine terms in Eq. 2 can be represented as,

$$
\begin{aligned}
& \vec{k}_{1 x} \cdot \vec{x}_{1}=\sigma k x, \quad \vec{k}_{1 y} \cdot \vec{y}_{1}=k y \\
& \vec{k}_{2 x} \cdot \vec{x}_{2}=\rho k(x \cos \alpha+y \sin \alpha), \quad \vec{k}_{2 y} \cdot \vec{y}_{2}=\rho k(-x \sin \alpha+y \cos \alpha)
\end{aligned}
$$

where $k$ is a wave number defined by $2 \pi / p_{y}$ ( $p_{y}$ is the height of the elemental optics) and $\alpha$ the superposing angle. The $\alpha$ does not exceed $\tan ^{-1}(1 / \sigma)$ because this angle indicates that the angle between the horizontal direction of the display panel and the vertical direction of elemental optics is $90^{\circ}-\alpha>45^{\circ}$. Hence $\alpha$ is in the range of $0 \leq \alpha \leq \tan ^{-1}(1 / \sigma)$. In Eq. 3, it is assumed that the height of each pixel is not less than the width of the pixel, the shapes of both pixel and elemental optics are the same but the size of the elemental optics is not smaller than that of the pixel. This means that the pixel and the elemental optics are in the form of a vertically elongated rectangle. By substituting Eq. 3 to Eq. 2, Eq. 3 is expressed as

$$
\begin{aligned}
& F=\frac{1}{16}\left[\begin{array}{l}
1+\cos \sigma k x+\cos k y+ \\
+\cos (\rho \cos \alpha k x+\rho \sin \alpha k y)+\cos (\rho \sin \alpha k x-\rho \cos \alpha k y)
\end{array}\right]+ \\
& \frac{1}{32}\left[\begin{array}{l}
\cos (\sigma k x+k y)+\cos (\sigma k x-k y)+ \\
+\cos (\rho(\cos \alpha-\sin \alpha) k x+\rho(\sin \alpha+\cos \alpha) k y)+ \\
+\cos (\rho(\cos \alpha+\sin \alpha) k x-\rho(\cos \alpha-\sin \alpha) k y)+ \\
+\cos ((\sigma+\rho \cos \alpha) k x+\rho \sin \alpha k y)+\cos ((\sigma-\rho \cos \alpha) k x-\rho \sin \alpha k y)+ \\
+\cos (\rho \cos \alpha k x+(1+\rho \sin \alpha) k y)+\cos (\rho \cos \alpha k x-(1-\rho \sin \alpha) k y)+ \\
+\cos ((\sigma-\rho \sin \alpha) k x+\rho \cos \alpha k y)+\cos ((\sigma+\rho \sin \alpha) k x-\rho \cos \alpha k y)+ \\
+\cos (\rho \sin \alpha k x+(1-\rho \cos \alpha) k y)+\cos (\rho \sin \alpha k x-(1+\rho \cos \alpha) k y)
\end{array}\right]+ \\
& \frac{1}{64}\left[\begin{array}{l}
\cos ((\sigma+\rho \cos \alpha) k x+(1+\rho \sin \alpha) k y)+\cos ((\sigma-\rho \cos \alpha) k x+(1-\rho \sin \alpha) k y)+ \\
+\cos ((\sigma+\rho \cos \alpha) k x-(1-\rho \sin \alpha) k y)+\cos ((\sigma-\rho \cos \alpha) k x-(1+\rho \sin \alpha) k y)+ \\
+\cos ((\sigma-\rho \sin \alpha) k x+(1+\rho \cos \alpha) k y)+\cos ((\sigma+\rho \sin \alpha) k x+(1-\rho \cos \alpha) k y)+ \\
+\cos ((\sigma-\rho \sin \alpha) k x-(1-\rho \cos \alpha) k y)+\cos ((\sigma+\rho \sin \alpha) k x-(1+\rho \cos \alpha) k y)+ \\
+\cos ((\sigma+\rho(\cos \alpha-\sin \alpha)) k x+\rho(\sin \alpha+\cos \alpha) k y)+ \\
+\cos (-\rho(\cos \alpha-\sin \alpha) k x+(1-\rho(\sin \alpha+\cos \alpha)) k y)+ \\
+\cos ((\sigma+\rho(\sin \alpha+\cos \alpha)) k x-\rho(\cos \alpha-\sin \alpha) k y)+ \\
+\cos ((\sigma-(\rho \sin \alpha+\cos \alpha)) k x+\rho(\cos \alpha-\sin \alpha) k y)+ \\
+\cos (\rho(\sin \alpha+\cos \alpha) k x+(1-\rho(\cos \alpha-\sin \alpha)) k y)+ \\
+\cos (\rho(\sin \alpha+\cos \alpha) k x-(1+\rho(\cos \alpha-\sin \alpha)) k y)
\end{array}\right]+ \\
& \left.+\begin{array}{l}
\cos ((\sigma+\rho(\cos \alpha-\sin \alpha)) k x+(1+\rho(\sin \alpha+\cos \alpha)) k y)+ \\
+\cos ((\sigma-\rho(\cos \alpha-\sin \alpha)) k x+(1-\rho(\sin \alpha+\cos \alpha)) k y)+ \\
+\cos ((\sigma+\rho(\cos \alpha-\sin \alpha)) k x-(1-\rho(\sin \alpha+\cos \alpha)) k y)+ \\
+\cos ((\sigma-\rho(\cos \alpha-\sin \alpha)) k x-(1+\rho(\sin \alpha+\cos \alpha)) k y)+ \\
+\cos ((\sigma+\rho(\sin \alpha+\cos \alpha)) k x+(1-\rho(\cos \alpha-\sin \alpha)) k y)+ \\
+\cos ((\sigma-\rho(\sin \alpha+\cos \alpha)) k x+(1+\rho(\cos \alpha-\sin \alpha)) k y)+ \\
+\cos ((\sigma+\rho(\sin \alpha+\cos \alpha)) k x-(1+\rho(\cos \alpha-\sin \alpha)) k y)+ \\
+\cos ((\sigma-\rho(\sin \alpha+\cos \alpha)) k x-(1-\rho(\cos \alpha-\sin \alpha)) k y)
\end{array}\right]
\end{aligned}
$$

Eq. 4 consists of 41 terms. It can be simplified by introducing the following relationships,

$$
\begin{aligned}
\cos (a k x+b k y) & =\cos \left\{\sqrt{a^{2}+b^{2}}\left(\frac{a}{\sqrt{a^{2}+b^{2}}} k x+\frac{b}{\sqrt{a^{2}+b^{2}}} k y\right)\right\} \\
& =\cos \left\{\sqrt{a^{2}+b^{2}}(\cos \varphi k x+\sin \varphi k y)\right\}
\end{aligned}
$$

and

$$
x^{\prime}=x \cos \varphi+y \sin \varphi
$$$$
y^{\prime}=-x \sin \varphi+y \cos \varphi
$$ 
where $\varphi=\tan ^{-1}(b / a)$. Then Eq. 4 can be expressed as,

$$
F=\sum_{n=1}^{41} q_{n} \cos \left(\sqrt{f_{n}(\rho, \alpha)} k x^{\prime}\left(y^{\prime}\right)\right)
$$

where $q_{n}$ is $1 / 16$ for $n=1$ to $5,1 / 32$ for $n=6$ to 17 , $1 / 64$ for $n=18$ to 33 and $1 / 128$ for $n=34$ to 41 , and $f_{1}(\rho, \alpha), f_{2}(\rho, \alpha), \ldots \ldots, f_{41}(\rho, \alpha)$ are given as, $0, \sigma_{: x}, 1_{: y}$,

$\rho_{: x^{\prime}}, \rho_{: y^{\prime}},\left(\sigma^{2}+1\right)_{: x^{\prime}},\left(\sigma^{2}+1\right)_{: y^{\prime}}, 2 \rho_{: x^{\prime}}, 2 \rho_{: y^{\prime}}$,

$\left(\sigma^{2}+2 \sigma \rho \cos \alpha+\rho^{2}\right)_{x^{\prime}}, \quad\left(\sigma^{2}-2 \sigma \rho \cos \alpha+\rho^{2}\right)_{y^{\prime}}$,

$\left(1+2 \rho \sin \alpha+\rho^{2}\right)_{: x^{\prime}}, \quad\left(1-2 \rho \sin \alpha+\rho^{2}\right)_{: y^{\prime}}$,

$\left(\sigma^{2}-2 \sigma \rho \sin \alpha+\rho^{2}\right)_{: x^{\prime}\left(y^{\prime}\right)},\left(\sigma^{2}+2 \sigma \rho \sin \alpha+\rho^{2}\right)_{: y^{\prime}}$,

$\left(1-2 \rho \cos \alpha+\rho^{2}\right)_{: x^{\prime}\left(y^{\prime}\right)}, \quad\left(1+2 \rho \cos \alpha+\rho^{2}\right)_{: y^{\prime}}$,

$\left\{\sigma^{2}+\rho^{2}+2 \rho(\sigma \cos \alpha+\sin \alpha)+1\right\}_{x^{\prime}}$,

$\left\{\sigma^{2}+\rho^{2}-2 \rho(\sigma \cos \alpha+\sin \alpha)+1\right\}_{x^{\prime}\left(y^{\prime}\right)}$,

$\left\{\sigma^{2}+\rho^{2}+2 \rho(\sigma \cos \alpha-\sin \alpha)+1\right\}_{:^{\prime}\left(x^{\prime}\right)}$,

$\left\{\sigma^{2}+\rho^{2}-2 \rho(\sigma \cos \alpha-\sin \alpha)+1\right\}_{:^{\prime}\left(x^{\prime}\right)}$,

$\left\{\sigma^{2}+\rho^{2}-2 \rho(\sigma \sin \alpha-\cos \alpha)+1\right\}_{x^{\prime}\left(y^{\prime}\right)}$,

$\left\{\sigma^{2}+\rho^{2}+2 \rho(\sigma \sin \alpha-\cos \alpha)+1\right\}_{x^{\prime}\left(y^{\prime}\right)}$,

$\left\{\sigma^{2}+\rho^{2}-2 \rho(\sigma \sin \alpha+\cos \alpha)+1\right\}_{: y^{\prime}\left(x^{\prime}\right)}$,

$\left\{\sigma^{2}+\rho^{2}+2 \rho(\sigma \sin \alpha+\cos \alpha)+1\right\}_{: y^{\prime}}$,

$\left\{\sigma^{2}+2 \rho^{2}+2 \sigma \rho(\cos \alpha+\sin \alpha)+1\right\}_{x^{\prime}}$,

$\left\{\sigma^{2}+2 \rho^{2}-2 \sigma \rho(\cos \alpha+\sin \alpha)+1\right\}_{: y^{\prime}\left(x^{\prime}\right)}$,

$\left\{\sigma^{2}+2 \rho^{2}+2 \sigma \rho(\cos \alpha-\sin \alpha)+1\right\}_{: x^{\prime}}$,

$\left\{\sigma^{2}+2 \rho^{2}-2 \sigma \rho(\cos \alpha-\sin \alpha)+1\right\}_{x^{\prime}}$,

$\left\{1+2 \rho^{2}+2 \rho(\sin \alpha+\cos \alpha)\right\}_{y^{\prime}}$,

$\left\{1+2 \rho^{2}-2 \rho(\sin \alpha+\cos \alpha)\right\}_{x^{\prime}\left(y^{\prime}\right)}$,

$\left\{1+2 \rho^{2}-2 \rho(\sin \alpha-\cos \alpha)\right\}_{x^{\prime}\left(y^{\prime}\right)}$,

$\left\{1+2 \rho^{2}+2 \rho(\sin \alpha-\cos \alpha)\right\}_{: y^{\prime}}$,

$\left\{1+\sigma^{2}+2 \rho^{2}-2 \rho((\sin \alpha-\cos \alpha) \sigma-(\sin \alpha+\cos \alpha))\right\}_{x^{\prime}}$,

$\left\{1+\sigma^{2}+2 \rho^{2}+2 \rho((\sin \alpha-\cos \alpha) \sigma-(\sin \alpha+\cos \alpha))\right\}_{: y^{\prime}\left(x^{\prime}\right)}$,

$\left\{1+\sigma^{2}+2 \rho^{2}-2 \rho((\sin \alpha-\cos \alpha) \sigma+(\sin \alpha+\cos \alpha))\right\}_{x^{\prime}}$,

$\left\{1+\sigma^{2}+2 \rho^{2}+2 \rho((\sin \alpha-\cos \alpha) \sigma+(\sin \alpha+\cos \alpha))\right\}_{:^{\prime}\left(x^{\prime}\right)}$,

$\left\{1+\sigma^{2}+2 \rho^{2}+2 \rho((\sin \alpha+\cos \alpha) \sigma-(\cos \alpha-\sin \alpha))\right\}_{:^{\prime}\left(y^{\prime}\right)}$,

$\left\{1+\sigma^{2}+2 \rho^{2}-2 \rho((\sin \alpha+\cos \alpha) \sigma-(\cos \alpha-\sin \alpha))\right\}_{: y^{\prime}}$,

$\left\{1+\sigma^{2}+2 \rho^{2}+2 \rho((\sin \alpha+\cos \alpha) \sigma+(\cos \alpha-\sin \alpha))\right\}_{: y^{\prime}}$,

and

$\left\{1+\sigma^{2}+2 \rho^{2}-2 \rho((\sin \alpha+\cos \alpha) \sigma+(\cos \alpha-\sin \alpha))\right\}_{: y^{\prime}\left(x^{\prime}\right)}$, respectively.
The order of these $f_{n}(\rho, \alpha)$ values corresponds to that of the cosine functions in Eq. 4.

\section{MINIMUM VALUE OF $f_{n}(\rho, \alpha)$}

In Eq. 6, the aspect ratio $\sigma$ can have only constant values and also, since $k=2 \pi / p_{y}, \sqrt{f_{n}(\rho, \alpha)} k=2 \pi /\left(p_{y}\right)$ $\left.\sqrt{f_{n}(\rho, \alpha)}\right)$. This means that the periods of moirés are $1 / \sqrt{f_{n}(\rho, \alpha)}$ times that of $p_{y}$ (the height of the elemental optics). I.e., as $f_{n}(\rho, \alpha)$ values decreases, the period of moirés increases. Hence it is possible to estimate the highest period of the moirés by selecting the lowest value of $f_{n}(\rho, \alpha)$ from the 41 values and finding the condition to minimize $f_{n}(\rho, \alpha)$. In this case, the periods of other moirés from the remaining $40 f_{n}(\rho, \alpha)$ values are below the highest one. If this highest period moiré can be made unrecognizable, the moirés become invisible.

According to Eq. 5, the moire will appear along $x$ and $y$, and $x^{\prime}$ and $y^{\prime}$ directions. However, the visible effect of the moirés in different directions will not be different. The most cumbersome and recognizable moirés are those changing their directions and periods depending on the viewing directions and distances. So it is necessary to classify the $f_{n}(\rho, \alpha)$ values into two groups of changing and unchanging directions. This grouping can be performed by Eqs. 4 and 5. From Eq. 4, $x^{\prime}$ and $y^{\prime}$ direction moirés can be identified by the $x$ and $y$ signs in the cosine terms because Eq. 5 informs us that when $x$ and $y$ have the same sign, it generates $x^{\prime}$ direction moirés, but different sign $y^{\prime}$ direction moirés. Among the 41 $f_{n}(\rho, \alpha)$ values, 0 represents no moiré, $\sigma$ and 1 represent moirés with the same period as the elemental optics, 11 and 12 of them are aligned in the $x^{\prime}$ and $y^{\prime}$ directions, respectively, and 15 of them change their directions depending on $\rho$ and $\sigma$ values. These are identified by the subscripts on $f_{n}(\rho, \alpha)$ values. From this grouping, it is not difficult to identify that the minimum value of the $f_{n}(\rho, \alpha)$ for the fixed and changing directions are $\sigma^{2}-2 \rho \sigma \cos \alpha+\rho^{2}$ and $\sigma^{2}+\rho^{2}-2 \sigma \rho \cos \alpha-$ $2 \rho \sin \alpha+1$, respectively, because $\cos \alpha>\sin \alpha$ for the given $\alpha$ range. Hence the moiré minimization condition can be found from these two functions. For convenience, let both functions by,

$$
\begin{aligned}
& f_{c g}(\rho, \alpha)=\sigma^{2}+\rho^{2}-2 \sigma \rho \cos \alpha-2 \rho \sin \alpha+1 \\
& f_{\text {ucg }}(\rho, \alpha)=\sigma^{2}+\rho^{2}-2 \sigma \rho \cos \alpha
\end{aligned}
$$

The conditions of minimizing these two functions are found by differentiating these functions by $\rho$ and $\alpha$. The partial derivative of Eq. 7 by $\rho$ and $\alpha$ results, 


$$
\begin{aligned}
& \frac{\partial}{\partial \rho} f_{c g}(\rho, \alpha)=2 \rho-2 \sigma \cos \alpha-2 \sin \alpha=0 \\
& \frac{\partial}{\partial \rho} f_{u c g}(\rho, \alpha)=2 \rho-2 \sigma \cos \alpha=0 \\
& \frac{\partial}{\partial \alpha} f_{c g}(\rho, \alpha)=2 \sigma \rho \sin \alpha-2 \rho \cos \alpha=0 \\
& \frac{\partial}{\partial \alpha} f_{u c g}(\rho, \alpha)=2 \sigma \rho \sin \alpha=0
\end{aligned}
$$

If the extreme values of $\rho$ and $\alpha$ for $f_{c g}(\rho, \alpha)$ and $f_{u c g}(\rho, \alpha)$ are set to $\rho_{c g}$ and $\sigma_{c g}$, and $\rho_{u c g}$ and $\sigma_{u c g}$, respectively, they are expressed as,

$$
\begin{aligned}
& \rho_{u c g}=\sigma \cos \alpha \quad \text { and } \quad \sin \alpha_{u c g}=0 \\
& \rho_{c g}=\sigma \cos \alpha+\sin \alpha \quad \text { and } \quad \tan \alpha_{c g}=\frac{1}{\sigma}
\end{aligned}
$$

Eq. 10 indicates that $f_{u c g}(\rho, \alpha)$ has the minimum when $\alpha=0$ and $f_{c g}(\rho, \alpha)$ when. $\alpha=\tan ^{-1} \frac{1}{\sigma}$. This means that within the domain of $\alpha, f_{u c g}(\rho, \alpha)$ and $f_{c g}(\rho, \alpha)$ show an opposite behavior because the value of $f_{\text {ucg }}(\rho, \alpha)$ will increase as $\alpha$ increases but that of $f_{c g}(\rho, \alpha)$ will decrease with increasing $\alpha$. Hence it is necessary to find an optimum value of $\alpha$ which makes $f_{\text {ucg }}(\rho, \alpha)=f_{c g}(\rho, \alpha)$ for the $\rho$ values which are minimizing each of $f_{u c g}(\rho, \alpha)$ and $f_{c g}(\rho, \alpha)$. However, for the case of $\rho_{\text {ucg }}, \rho_{\text {ucg }}=\sigma \cos \alpha$ does not comply with the condition $\rho \geq \sigma$, except $\rho_{\text {ucg }}=\sigma$ for the given $\alpha$ domain, i.e., $0 \leq \alpha \leq \tan ^{-1}(1 / \sigma)$. So $\rho_{u c g}$ should be $\sigma$ only. By substituting $\rho_{u c g}=\sigma$ and $\rho_{c g}=\sigma \cos \alpha+\sin \alpha$ into $f_{u c g}(\rho, \alpha)$ and $f_{c g}(\rho, \alpha)$, respectively, and making them equal, the following relationship is obtained.

$$
\sigma^{2}(1-2 \cos \alpha)=\left(1-\sigma^{2}\right) \cos ^{2} \alpha-2 \sigma \cos \alpha \sin \alpha
$$

When $\cos \alpha$ is replaced by $u$, Eq. 11 will be rewritten as,

$$
\left(1+\sigma^{2}\right)^{2} u^{4}+4 \sigma^{2}\left(1-\sigma^{2}\right) u^{3}+6 \sigma^{2}\left(\sigma^{2}-1\right) u^{2}-4 \sigma^{4} u+\sigma^{4}=0
$$

When $u$ is solved with MATHCAD version 14 for $\sigma$ values of $1.0,1.5,2.0,2.5,3.0,3.5$ and 4.0 , the angles corresponding to these $\sigma$ values are calculated as, $26.2609^{\circ}, 18.3245^{\circ}, 13.9858^{\circ}, 11.283^{\circ}, 9.4465^{\circ}, 8.12^{\circ}$ and $7.1182^{\circ}$, respectively. These are the superposing angles between the viewing zone forming optics and the display panel which are chosen to minimize the moirés. The angle $26.2609 \mathrm{fl}$ for $\sigma=1$, i.e., a square grid case, had been demonstrated as giving no visible moirés[6].

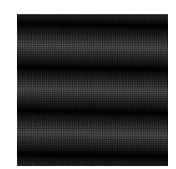

$\alpha=2^{\circ}$

Unfiltered

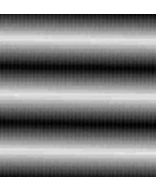

$\alpha=2^{\circ}$

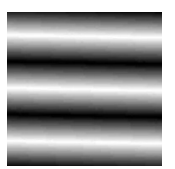

$\alpha=2^{\circ}$

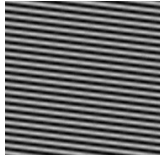

$\alpha=14^{\circ}$

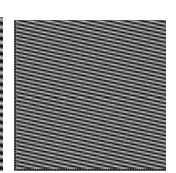

$\alpha=26.57^{\circ}$

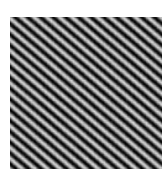

$\alpha=36^{\circ}$

$$
\sigma=1 \text { and } \rho=1
$$

FIG. 1. Moires calculated from Eq. 1.

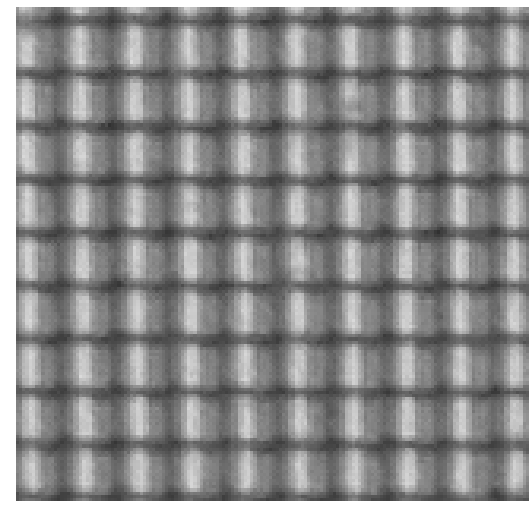

Samsung Syne Master

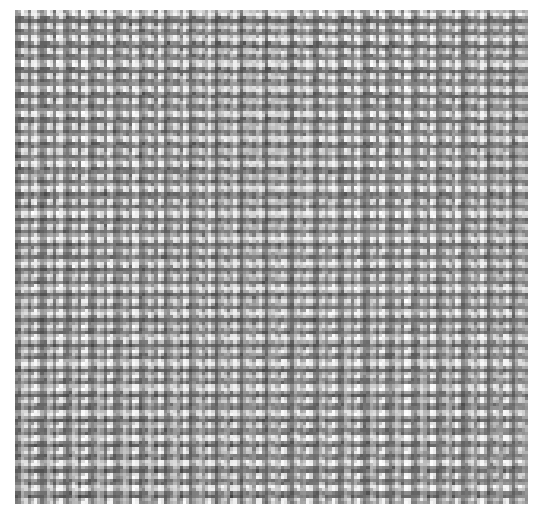

IBM

FIG. 2. Comparison of pixel patterns of Samsung and IBM monitors

\section{EXPERIMENTAL RESULTS}

Fig. 1 shows moirés calculated from Eq. 4 by assuming for comparison that $\rho=1$ for both $\sigma=1$ and 2. The $\alpha$ angles used are $2^{\circ}, 14^{\circ}, 26.57^{\circ}$ and $36^{\circ}$ for $\sigma=1$, and $2^{\circ}, 7^{\circ}, 14^{\circ}$ and $21^{\circ}$ for $\sigma=2$.

In Fig. $1,2^{\circ}$ is used because $0^{\circ}$ shows very unstable 
characteristics. Furthermore, high frequency components are eliminated to show the behavior of lowest frequency (longest period) components. Fig. 1 shows that the moire periods for the angle $26.57^{\circ}$ (This angle corresponds to two pixel height for the square pixel) and $14^{\circ}$ are the smallest for the $\sigma=1$ and 2 cases, respectively.

The effectiveness of the angles in minimizing the moirés is experimentally demonstrated by using a Samsung SyncMaster 177x composed of an array of square pixels and an IBM 9503-DG3 (22 inch) an array of rectangular pixels with aspect ratio 2. Fig 2 shows the magnified pixel patterns of Samsung and IBM when a white field is displayed.

The pixel pattern of the Samsung is characterized by square pixels. Each of the pixels is subdivided vertically into 3 primary color sub-pixels. For the IBM, the pattern is also characterized by square pixels. And each of the pixels is subdivided into sub-pixels, however, each square pixel is additionally subdivided into two halves by a horizontal line. This makes the IBM monitor behave as if it had rectangular pixels with aspect ratio 2. The pixel pitches of Samsung and IBM monitors are $0.264 \mathrm{~mm}$ and $0.1245 \mathrm{~mm}$, respectively. For the experiment, the lenticular plates with pitches of $1.075 \mathrm{~mm}$ and $0.34 \mathrm{~mm}$ are superposed on the Samsung and IBM monitors, respectively. The pitch values correspond to $\rho$ values of 4.07 and 2.73 , respectively. The superposing angle of the Samsung is $26.57^{\circ}$ and that of the IBM $14^{\circ}$. Fig. 3 shows the moires appearing on the monitors. The pitches of moirés are measured as $0.35 \mathrm{~mm}$ and $0.34 \mathrm{~mm}$ for Samsung and IBM monitors. The moiré pitches are almost the same. However, the pitches are corresponding to 1.33 pixels and 2.73 pixels in their corresponding monitors. These period moirés are hardly recognized by viewers'eyes at the nominal viewing distance of the monitors because of the average human eyes'resolution limit which is estimated as $1 \mathrm{~mm}$ at $1.5 \mathrm{~m}[9]$. It is noticed that the moire pitch values are slightly different from the values calculated from $\sqrt{f_{u c g}(\rho, \alpha)}$. The pitch values calculated for the Samsung and IBM are $0.335 \mathrm{~mm}$ and $3.7 \mathrm{~mm}$, respectively. The difference is probably caused by the alignment errors. It is also noticed that as shown in Eq. 11, the $\alpha$ angle for minimizing moirés doesn't depend on $\rho$, i.e., it keeps the same value without regard to $\rho$ values.

\section{CONCLUSIONS}

There are two major types of moirés which appear at contact-type 3 dimensional imaging systems. One keeps the same directions and another changing with changes parameter values. From each of these two

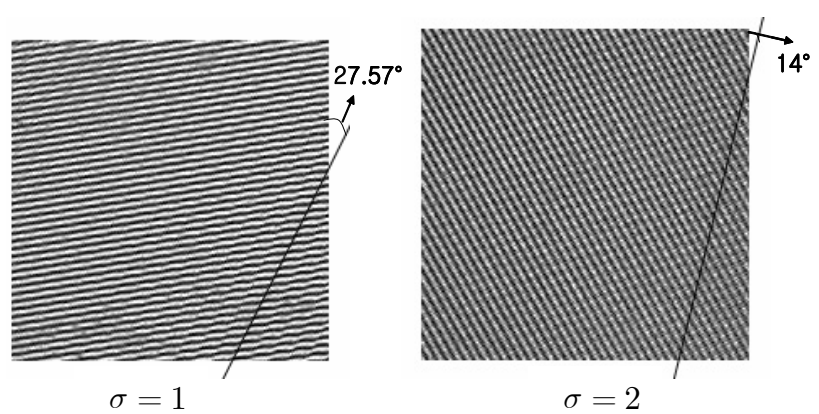

FIG. 3. Moires appearing on Samsung Syncmaster 177x $(\sigma=2)$ and IBM 9503-DG3 $(\sigma=2)$ Monitors

types, the moire with the highest period moire is selected and the periods made equal to each other to calculate the moire minimizing angle. The angle is $26.261^{\circ}$ for the square shaped pixels, and $13.98^{\circ}$ for the rectangular shape pixels with aspect ratio 2 . It is experimentally proved that these angles produce the smallest period moirés.

\section{REFERENCES}

[1] I. Amidror, The Theory of the Moire Phenomenon, Cluver Academic Publishers, pp. 28-69, 2000.

[2] T. Yamada and S. Yokozeki, Measurement Methods Using Moire Techniques and/or Interferometry (Japanese Edition), Corona Publishing Co, Tokyo, Japan, ch.2, pp. 19-44, 1996 .

[3] Jung-Young Son and Bahram Javidi, "3-Dimensional Imaging Systems Based on Multiview Images," IEEE/ OSA J. of Display Technology, Vol. 1, no 1, pp. 125140, 2005.

[4] Jung-Young Son, Bahram Javidi and Kae-Dal Kwack, "Methods for Displaying 3 Dimensional Images," Proceedings of the IEEE, Special Issue on: 3-D Technologies for Imaging \& Display, Vol. 94, No. 3, pp. 502-523, 2006.

[5] Jung-Young Son, V. V. Saveljev and Bahram Javidi, "3 dimensional images with no viewing zone forming optics," Sixth Euro-American Workshop on Information Optics 2007(WIO'07), AIP Conference Proceedings no. 949, pp. 121-129, 2007.

[6] Vladmir V. Saveljev, Jung-Young Son, Bahram Javidi, Sung-Kyu Kim and Dae-Sik Kim, "A Moiré Minimization Condition in 3 Dimensional Image Displays," J. of Display Technology, Vol. 1, no. 2, pp. 347-353, 2005.

[7] J.-Y. Son, V. Saveljev, K.-T. Kim, and S.-S. Kim, Minimizing Moire Fringes in Contact- Type 3-Dimensional Imaging Systems, Proc. 17th Annual Meeting of the IEEE Laser \& Electro-Optics Society (LEOS'2004), pp. 350-351, 2004.

[8] www-03.ibm.com

[9] V. G. Komar, and O. B. Serov, Art Holography and Holographic Cinematograthy (Iskusstvo, Moscow, 1990). 